\title{
Pregnancy with and without birth trauma modulates the gene expression of proteins involved in elastic fiber homeostasis in the rat vagina
}

\author{
K. T. Downing ${ }^{*}$, F. A. Strube ${ }^{1}$, M. S. Mikhail ${ }^{2}$, M. E. DiSanto ${ }^{3}$ \\ ${ }^{1}$ Department of Obstetrics, Gynecology \& Women's Health, Albert Einstein College of Medicine-Montefiore Medical Center, New \\ York, USA \\ ${ }^{2}$ Obstetrics and Gynecology, Bronx Lebanon Hospital Center, New York, USA \\ ${ }^{3}$ Department of Biomedical Sciences, Cooper University Hospital \& Cooper Medical School of Rowan University, Camden, USA \\ Email: ${ }^{*}$ kdowning@montefiore.org
}

Received 26 June 2013; revised 25 July 2013; accepted 2 August 2013

Copyright (C) 2013 K. T. Downing et al. This is an open access article distributed under the Creative Commons Attribution License, which permits unrestricted use, distribution, and reproduction in any medium, provided the original work is properly cited.

\begin{abstract}
Objectives: Elastic fibers have been linked to the pathogenesis of pelvic organ prolapse. This study used a rat model to examine the effect of mode of delivery on the gene expression of proteins involved in elastic fiber homeostasis. Methods: 38 Sprague-Dawley rats were separated as follows: virgin rats after simulated vaginal delivery, groups $A(n=5)$ and $B(n=$ 5); pregnant rats after spontaneous vaginal delivery, groups $C(n=5)$ and $D(n=5)$; pregnant rats after cesarean delivery, groups $E(n=6)$ and $F(n=5)$. Groups $A, C$ and $E$ were sacrificed 2 days after the intervention, groups $B, D$ and $F$ were sacrificed 14 days after the intervention. 7 virgin rats served as controls. Vaginal tissue was harvested for RNA extraction and cDNA conversion. The mRNA expression for lysyl oxidase like 1 (LOXL-1), tropoelastin and fibulin 5 (FBLN 5) was quantified via real-time reverse transcriptase polymerase chain reaction using the $2^{-\Delta \Delta C t}$ method; $\boldsymbol{\beta}$-actin was selected as the reference gene. Results: Postpartum day two we observed an up-regulation in the relative $\mathrm{mRNA}$ expression of LOXL-1, FBLN 5 and Tropoelastin in all groups compared to controls. In lower vaginal tissue the up-regulation decreased uniformly from postpartum day two to postpartum day fourteen. However, the relative mRNA expression of the target genes in upper vaginal tissue persisted at postpartum day fourteen in the SpVD and CD cohort. Conclusions: Vaginal trauma, whether as a result of spontaneous vaginal delivery or simulated vaginal delivery, resulted in the upregulation of genes involved in elastic fiber
\end{abstract}

\footnotetext{
*Corresponding author.
}

homeostasis. In addition, pregnancy and parturition, in the absence of direct vaginal trauma, also resulted in the upregulation of these genes. Our findings leave open the possibility that alterations in elastic fiber homeostasis occurring during pregnancy, regardless of mode of delivery, may play a role in the pathogenesis of POP.

Keywords: Elastic Fiber Homeostasis; Pregnancy

\section{INTRODUCTION}

Pelvic organ prolapse (POP) is a major health and quality of life problem that affects women in their reproductive and menopausal years [1]. The lifetime risk of having surgery for POP is as high as $19 \%$, while the annual cost to surgically manage POP in the U.S. is estimated to be $\$ 1$ billion [2,3]. The causes of POP common to most women include pregnancy and parturition, and aging. With respect to the former, increasing vaginal parity, and operative vaginal delivery have been identified as the exposures most associated with higher rates of POP; yet, most women who have born children do not develop POP and when they do so it is well after the associated exposure [4-7]. Our inability to explicate this discrepancy, in part, relates to our limited understanding of the pathophysiology of POP.

In recent years a significant advancement in our understanding of the pathophysiology of POP came to light when an important physiologic link between elastic fiber homeostasis and POP was observed in two murine knockout (KO) models (Lysyl Oxidase Like-1 [LOXL-1] and Fibulin 5 [FBLN 5]) that develop POP due to abnormalities in elastic fiber formation $[8,9]$. Elastic fibers consist 
of two distinct components: elastin and microfibrils. Elastin is an insoluble polymer that consists of crosslinked tropoelastin monomers, while microfibrils are made up of a complex array of macromolecules [10]. LOXL-1 is thought to play a role in the cross-linking of tropoelastin monomers (elastin's precursor) to create elastin polymers, while FBLN 5 is thought to play a role in the transfer of elastin aggregates to microfibrils, the microfibrils acting as the scaffolding of elastic fibers [11]. Elastic fibers are found throughout the extracellular matrix of the reproductive tract and endow these tissues with the characteristic of elastic recoil [12]. While elastic fibers in most tissues undergo little change during adult life, those of the reproductive tract differ by undergoing significant remodeling as a result of pregnancy and parturition [13]. Recent studies have illuminated this process. Drewes and colleagues studied the regulation of elastic fiber synthesis and assembly in the vaginal wall and pubocaudalis tissues of wild type mice. They found a burst in the protein expression of FBLN 5, tropoelastin and desmosine (desmosine is a marker of mature elastic fibers), during the immediate postpartum period. This led them to hypothesize that this burst in elastic fiber synthesis and assembly was critical to the recovery of pelvic floor support after vaginal delivery, and its absence or derangement may play a primary role in the pathogenesis of POP. To test this hypothesis, Drewes and colleagues injected the posterior vaginal wall of wild type mice with elastase immediately postpartum, and found POP developed in the elastase injected mice, but not in saline or inactivated elastase treated animals [14].

To test the hypothesis posited by Drewes, Rahn and colleagues exposed nulliparous FBLN 5 KO, FBLN 5 heterogeneous, and wild type mice to vaginal distention (to simulate birth trauma) at 6 weeks of age (prior to the spontaneous onset of prolapse in FBLN 5 KO mice) and found that within days of the exposure only the FBLN 5 KOs developed pelvic organ prolapse [15].

In light of these findings, our group, using a rat model, has examined the effect of pregnancy and mode of delivery on the gene expression of proteins involved in elastic fiber synthesis and assembly.

\section{MATERIALS AND METHODS}

All chemicals were purchased from Sigma Chemicals (St. Louis, MO, USA) except as noted below. TRIzol reagent, oligo (dT) and the SuperScript II First-Strand Synthesis System for reverse transcriptase-PCR (RT-PCR) were from Invitrogen Corporation (Carlsbad, CA, USA). SYBR $^{\circledR}$ Green PCR Master Mix and 96-well PCR plates/ covers were from Applied Biosystems Inc. (Foster City, CA, USA). Primers were synthesized by Invitrogen Corporation and the sequences for the individual molecular targets are listed in Table $\mathbf{1 .}$
All study protocols were approved by the Animal Institute Committee of the Albert Einstein College of Medicine. The study used 38 female Sprague-Dawley rats. 10 virgin rats underwent simulated vaginal delivery (SiVD) as follows: under anesthesia a 10 French Foley catheter was inserted into the vagina, the Foley balloon inflated with $\sim 2.5 \mathrm{cc}$ of water, and the catheter placed to gravity off the edge of the lab bench for 3 hours, then removed while still inflated. 10 pregnant rats underwent spontaneous vaginal delivery (SpVD). 11 pregnant rats were submitted to cesarean delivery (CD) on gestation day 21 as follows: under anesthesia, a small vertical incision was made in the lower ventral abdominal wall allowing for delivery of the gravid uterine horns. Through longitudinal incisions, rat pups and their placentas were removed; the horns were returned to the abdominal cavity and the ventral incision closed with permanent suture. Experimental animals were sacrificed on day 2 or day 14 after their respective mode of delivery and categorized as shown in Figure 1. 7 virgin rats served as controls.

All rats were killed humanely by placing them in a

Table 1. Summary of target genes and the primer sequences used to amplify them in real-time PCR.

\begin{tabular}{|c|c|}
\hline$\underline{\text { Target Gene }}$ & Primer sequences, $5^{\prime}$ to $3^{\prime}$ \\
\hline LOXL-1 forward & GTGCAGCCTGGGAACTACATC \\
\hline LOXL-1 reverse & TGGTGAAGTCAGACTCCAGAACA \\
\hline Tropoelastin forward & TCCTAGGAGCCAGGCCATT \\
\hline Tropoelastin reverse & GGGTAGATAGGAGAAAGTCCAAACC \\
\hline FBLN 5 forward & TGCAAGCAACGACCCGATA \\
\hline FBLN 5 reverse & CCCTCGTTCCCAGATTTGATC \\
\hline Beta actin forward & CTTCAACACCCCAGCATGT \\
\hline Beta actin reverse & AGTGGTACGACCAGAGGCATACA \\
\hline $\begin{array}{l}\text { 10- Simulated Vaginal } \\
\text { Delivery (SiVD) }\end{array}$ & in Group B: Sacrifice Day 14 post SiVD \\
\hline $\begin{array}{l}\text { 10- Spontaneous Vaginal } \\
\text { Delivery (SpVD) }\end{array}$ & Iroup C: Sacrifice Day 2 post SpVD \\
\hline & Day 2 post CD \\
\hline
\end{tabular}

Figure 1. Group allocation based on mode of delivery and timing of postpartum sacrifice $* 7$ virgin rats were used as controls. 
carbon dioxide chamber. Immediately after sacrifice, vaginal tissue was harvested and divided into an upper segment (from the mid-vagina to the level of the vaginal fornices) and lower segment (from the mid-vagina to the level of the perineal body), and the tissue flash frozen.

Total RNA was isolated from the frozen vaginal tissue of each rat using TRIzol reagent according to the manufacturer's protocol. RNA concentration and purity was determined using a ND-1000 spectrophotometer (NanoDrop Technologies, Wilmington, DE, USA). For each sample, $1 \mu \mathrm{g}$ of RNA was converted to cDNA using RT via the SuperScript II First-Strand Synthesis System according to the manufacturer's protocol. Primer pairs were designed using published cDNA sequences obtained via Entrez Nucleotide of the National Center for Biotechnology Information and the Primer Express program (Applied Biosystems). Amplicons were deliberately kept between 50 and $100 \mathrm{bp}$ for all primer pairs to enable equal transcriptional efficiency. For RT-PCR, equal amounts of cDNA template and primer pairs were combined with SYBR Green Master Mix to final concentrations of $0.04-0.4 \mathrm{ng} / \mu \mathrm{L}$ and $50-900 \mathrm{~nm}$, respectively, depending on the molecular target, and real time RT-PCR performed as previously described [16]. The following targets were amplified using SYBR Green for amplicon detection: LOXL-1, Tropoelastin, FBLN 5. For relative quantification the efficiency of amplification for each individual primer pair was determined using cDNA target and the $2^{-\Delta \Delta \mathrm{ct}}$ method [17] in conjunction with the RQ Study Software version 1.2.3 (Applied Biosystems). Gene expression was normalized to expression of the beta actin reference gene.

\section{RESULTS}

On postpartum day two the relative mRNA expression of all three target genes in upper and lower vaginal tissue was found to be up-regulated when compared to controls (see Figures 2 and 3). By postpartum day fourteen the relative mRNA expression of the target genes in lower vaginal tissue had uniformly decreased from their day two levels (see Figure 3). In upper vaginal tissue this trend remained true for the SiVD cohort. However, in both pregnancy cohorts (SpVD and $\mathrm{CD}$ ), the relative mRNA expression of our target genes on day fourteen remained similar to day two expression levels, and in the case of LOXL-1 and FBLN 5, in the CD cohort, actually increased (see Figure 2). Finally, though our experimental methods were not designed to detect statistical difference between tissue origin, we observed that the relative mRNA expression of our target genes in the SiVD cohort was notably more up-regulated in lower vaginal tissue compared to upper vaginal tissue at postpartum day two (see Figures 2 and $\mathbf{3}$ ).

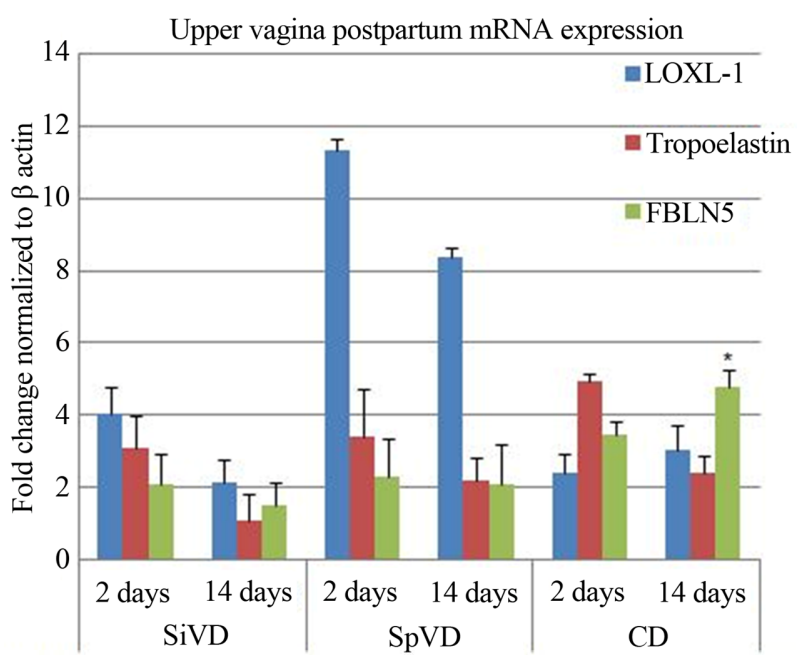

Figure 2. Bar graph representing the relative mRNA expression level of LOXL-1, Tropoelastin and FBLN5 at 2 days and 14 days after SiVD (Simulated Vaginal Delivery), SpVD (Spontaneous Vaginal Delivery) and CD (Cesarean Delivery) in upper vaginal tissue. The error bar represents the standard error measure and the asterisk the statistical significance at $\mathrm{P}<0.05$ compared to control (Student's t-test). Results are normalized to $\beta$ actin and set to a value of 1 for control.

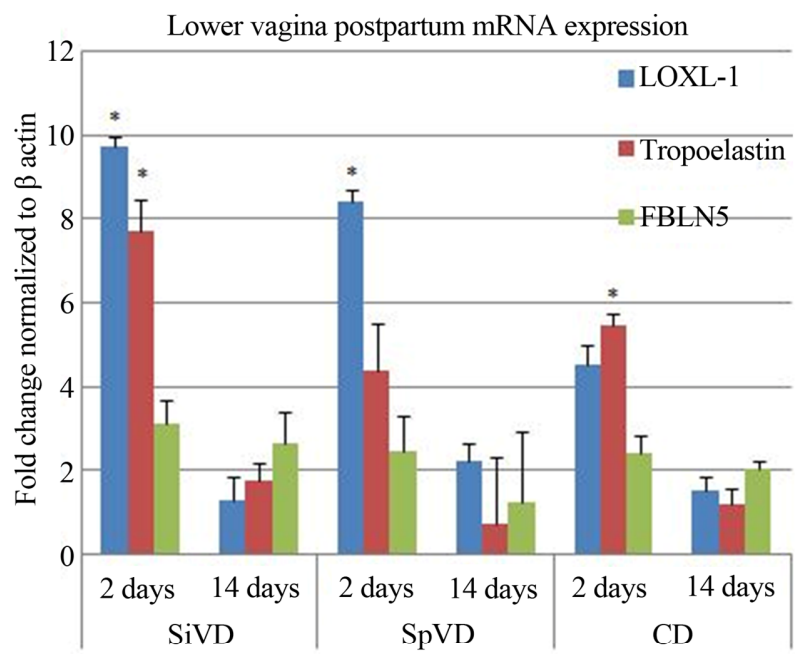

Figure 3. Bar graph representing the relative mRNA expression level of LOXL-1, Tropoelastin and FBLN5 at 2 days and 14 days after SiVD (Simulated Vaginal Delivery), SpVD (Spontaneous Vaginal Delivery) and CD (Cesarean Delivery) in lower vaginal tissue. The error bar represents the standard error measure and the asterisk the statistical significance at $\mathrm{P}<0.05$ compared to control (Student's t-test). Results are normalized to $\beta$ actin and set to a value of 1 for control.

\section{DISCUSSION}

Our understanding of the pathophysiology of POP and progress in its management has developed slowly over time [18]. Over the last decade, a number of studies have drawn an association between vaginal parity and pelvic floor disorders (PFDs) including POP [5-7,19,20]. These 
studies and others have caused some leaders in the field to address the question of whether there is a role in twenty-first century obstetrics for elective cesarean delivery for the primary purpose of preventing PFDs [21]. With the cesarean delivery rate over $30 \%$ in the United States, this might seem a reasonable option for patients and physicians, particularly in women who are older and desire low parity [22]. Yet, to the contrary, pregnancy alone has been found to be associated with changes in pelvic floor support and function, and cesarean delivery (even before labor) is not completely protective of PFDs [23-25]. Further, with advancing age, the rates of some PFDs in nulliparous women rival that of their parous counterparts [26].

One of the challenges to better understanding the relationship between pregnancy, mode of delivery and PFDs has been the lack of a suitable animal model. Non-human primates have been used to study pelvic floor disorders because of their similarity to humans in pelvic anatomy, the 28-day menstrual cycle, manner of parturition, and the onset of POP. In addition, non-human primates share two of the evolutionary factors associated with PFDs: semi-upright posture and giving birth to infants with large cranial capacity. Nevertheless, non-human primates (e.g., squirrel monkeys), have their limitations such as the inability to take instruction, report symptoms, or inform intentions. Most notably, they have a greater associated cost when compared to the use of murine models, resulting in limited use in published studies [27].

Although quadrupeds, the development of $\mathrm{KO}$ mice to LOXL-1 and FBLN 5 with their abnormalities in elastic fiber homeostasis have become important animal models to aid the study of the complex relationship between pregnancy, parturition, aging and POP. In addition to $\mathrm{KO}$ mice, the application of vaginal distention in murine models to mimic the effects of birth trauma on pelvic floor tissues has provided another valuable research tool. Use of these techniques has been best exemplified in the work of Drewes et al. and Rahn et al. [14,15]. Their results have affirmed the link between elastic fibers, vaginal delivery and POP in a murine model. However, there has only been one report analyzing the effect of cesarean delivery compared to vaginal delivery on elastic fiber homeostasis in pelvic floor tissues in a murine model [28]. In that study by Gustilo-Ashby et al., LOXL-1 KO mice were submitted to either spontaneous or cesarean delivery and had POP assessed at 12 weeks postpartum. They found $59 \%$ of LOXL-1 KO mice after spontaneous vaginal delivery and $31 \%$ after cesarean delivery developed POP, with those in the vaginal delivery group developing POP approximately three weeks earlier than those in the cesarean delivery group.

In our experiment we determined the relative mRNA expression (using real time RT PCR) of proteins responsible for elastic fiber synthesis and assembly in the early (day two) and late (day fourteen) postpartum period from vaginal tissue taken from Sprague Dawley rats that had been exposed to different modes of delivery. We observed an up-regulation in the relative mRNA expression of LOXL-1, FBLN 5 and Tropoelastin in all groups at postpartum day two. In lower vaginal tissue the up-regulation decreased uniformly from postpartum day two to postpartum day fourteen. However, the relative mRNA expression of the target genes in upper vaginal tissue persisted at postpartum day fourteen in the SpVD and CD cohort. This finding may reflect the influence of pregnancy on tissues of mullerian origin (as opposed to the urogenital sinus derived tissues of the lower vagina) and the postpartum involution process that is unrelated to tissue trauma. In addition, we observed little change in the relative mRNA expression of the target genes in upper vaginal tissue in the SiVD cohort. This may be a function of our experimental model, in which the foley balloon, due to its size and shape, distends and damages tissues of the lower vagina but spares the upper vault. We hope to clarify this finding by determining the extent of elastic fiber damage in the upper and lower vaginal vault after balloon distention by histologic examination.

Finally, to better understand the effect of mode of delivery on elastic fiber homeostasis in our model, we will need to analyze the enzyme activity of elastolytic enzymes such as matrix metalloproteinase 2 and 9 (MMP 2 and MMP 9). These elastolytic enzymes are found in the extracellular matrix of the reproductive tract and have been shown to be affected by pregnancy and parturition [29]. In an elegant experiment using a double KO mouse to FBLN 5 and MMP-9, Budatha et al., showed that the development of mouse POP not only required aberrant elastic fibers, but also the up-regulation of MMP-9 [30]. Thus, the effect of mode of delivery on MMP expression could have significant bearing on the development of POP.

\section{CONCLUSION}

In this study we found that vaginal trauma, whether by balloon distention or vaginal delivery results in the upregulation of genes involved in elastic fiber synthesis and assembly. Further, we found that pregnancy, in the absence of vaginal trauma (i.e., cesarean delivery), also resulted in the up-regulation of these genes. In total, these findings leave open the possibility that alterations in elastic fiber homeostasis occurring during pregnancy, regardless of mode of delivery, may play a role in the patho- genesis of POP and other pelvic floor disorders.

\section{REFERENCES}

[1] Nygaard, I., Barber, M.D., Burgio, K.L., et al. (2008) Prevalence of symptomatic pelvic floor disorders in US wo- 
men. The journal of the American Medical Association, 300, 1311-1316.

http://dx.doi.org/10.1001/jama.300.11.1311

[2] Smith, F.J., Holman, C.D., Moorin, R.E. and Tsokos, N. (2010) Lifetime risk of undergoing surgery for pelvic organ prolapse. Obstetrics and Gynecology, 116, 1096- 1100. http://dx.doi.org/10.1097/AOG.0b013e3181f73729

[3] Subak, L.L., Waetjen, L.E., van den Eeden, S., Thom, D.H., Vittinghoff, E. and Brown, J.S. (2001) Cost of pelvic organ prolapse surgery in the United States. Obstetrics and Gynecology, 98, 646-651.

http://dx.doi.org/10.1016/S0029-7844(01)01472-7

[4] Bump, R.C. and Norton, P.A. (1998) Epidemiology and natural history of pelvic floor dysfunction. Obstetrics and Gynecology Clinics of North America, 25, 723-746. http://dx.doi.org/10.1016/S0889-8545(05)70039-5

[5] Handa, V.L., Blomquist, J.L., Knoepp, L.R., Hoskey, K.A., McDermott, K.C. and Munoz, A. (2011) Pelvic floor disorders 5-10 years after vaginal or cesarean childbirth. $\mathrm{Ob}$ stetrics and Gynecology, 118, 777-784.

[6] Leijonhufvud, A., Lundholm, C., Cnattingius, S., Granath, F., Andolf, E. and Altman, D. (2011) Risks of stress urinary incontinence and pelvic organ prolapse surgery in relation to mode of childbirth. American Journal of $\mathrm{Ob}$ stetrics and Gynecology, 204, 70e 71-77.

[7] Lukacz, E.S., Lawrence, J.M., Contreras, R., Nager, C.W. and Luber, K.M. (2006) Parity, mode of delivery, and pelvic floor disorders. Obstetrics and Gynecology, 107, 12531260.

http://dx.doi.org/10.1097/01.AOG.0000218096.54169.34

[8] Liu, X., Zhao, Y., Gao, J., et al. (2004) Elastic fiber homeostasis requires lysyl oxidase-like 1 protein. Nature Genetics, 36, 178-182. http://dx.doi.org/10.1038/ng1297

[9] Yanagisawa, H., Davis, E.C., Starcher, B.C., et al. (2002) Fibulin-5 is an elastin-binding protein essential for elastic fibre development in vivo. Nature, 415, 168-171. http://dx.doi.org/10.1038/415168a

[10] Mecham RPaD, E.C. (1994) Elastic fiber structure and assembly, Academic Press, San Diego.

[11] Wagenseil, J.E. and Mecham, R.P. (2007) New insights into elastic fiber assembly. Birth Defects Research. Part C, Embryo Today: Reviews, 81, 229-240.

[12] Starcher, B. and Percival, S. (1985) Elastin turnover in the rat uterus. Connective Tissue Research, 13, 207-215. http://dx.doi.org/10.3109/03008208509152400

[13] Woessner, J.F. and Brewer, T.H. (1963) Formation and breakdown of collagen and elastin in the human uterus during pregnancy and post-partum involution. The Biochemical Journal, 89, 75-82.

[14] Drewes, P.G., Yanagisawa, H., Starcher, B., et al. (2007) Pelvic organ prolapse in fibulin-5 knockout mice: Pregnancy-induced changes in elastic fiber homeostasis in mouse vagina. The American Journal of Pathology, 170, 578-589. http://dx.doi.org/10.2353/ajpath.2007.060662

[15] Rahn, D.D., Acevedo, J.F. and Word, R.A. (2008) Effect of vaginal distention on elastic fiber synthesis and matrix degradation in the vaginal wall: Potential role in the pathogenesis of pelvic organ prolapse. American Journal of Physiology. Regulatory, Integrative and Comparative Physiology, 295.

http://dx.doi.org/10.1152/ajpregu.90447.2008

[16] Sandhu, K.S., Chua, R.G., Zhang, X., et al. (2009) Regional heterogeneity in expression of the sphingosine1-phosphate pathway in the female rat lower urinary tract. American Journal of Obstetrics and Gynecology, 200, 576 e $571-577$.

[17] Livak, K.J. and Schmittgen, T.D. (2001) Analysis of relative gene expression data using real-time quantitative PCR and the $2^{-\Delta \Delta \mathrm{ct}}$ method. Methods, 25, 402-408. http://dx.doi.org/10.1006/meth.2001.1262

[18] Downing, K.T. (2012) Uterine prolapse: From antiquity to today. Obstetrics and Gynecology International, 2012. http://dx.doi.org/10.1155/2012/649459

[19] Rortveit, G., Daltveit, A.K., Hannestad, Y.S., Hunskaar, S. (2003) Urinary incontinence after vaginal delivery or cesarean section. The New England Journal of Medicine, 348, 900-907. http://dx.doi.org/10.1056/NEJMoa021788

[20] Dietz, H.P. and Bennett, M.J. (2003) The effect of childbirth on pelvic organ mobility. Obstetrics and Gynecology, 102, 223-228. http://dx.doi.org/10.1016/S0029-7844(03)00476-9

[21] Nygaard, I. and Cruikshank, D.P. (2003) Should all women be offered elective cesarean delivery? Obstetrics and Gynecology, 102, 217-219. http://dx.doi.org/10.1016/S0029-7844(03)00603-3

[22] Martin, J.A., Hamilton, B.E., Ventura, S.J., et al. (2011) Births: Final data for 2009. National Vital Statistics Reports: From the Centers for Disease Control and Prevention, National Center for Health Statistics, National Vital Statistics System, 60, 1-70.

[23] O’Boyle, A.L., Woodman, P.J., O’Boyle, J.D., Davis, G.D. and Swift, S.E. (2002) Pelvic organ support in nulliparous pregnant and nonpregnant women: A case control study. American Journal of Obstetrics and Gynecology, 187, 99-102. http://dx.doi.org/10.1067/mob.2002.125734

[24] Hannah, M.E., Whyte, H., Hannah, W.J., et al. (2004) Maternal outcomes at 2 years after planned cesarean section versus planned vaginal birth for breech presentation at term: The international randomized Term Breech Trial. American Journal of Obstetrics and Gynecology, 191, 917-927. http://dx.doi.org/10.1016/j.ajog.2004.08.004

[25] Thorp Jr., J.M., Norton, P.A., Wall, L.L., Kuller, J.A., Eucker, B. and Wells, E. (1999) Urinary incontinence in pregnancy and the puerperium: A prospective study. American Journal of Obstetrics and Gynecology, 181, 266273. http://dx.doi.org/10.1016/S0002-9378(99)70546-6

[26] Buchsbaum, G.M., Chin, M., Glantz, C. and Guzick, D. (2002) Prevalence of urinary incontinence and associated risk factors in a cohort of nuns. Obstetrics and Gynecology, 100, 226-229. http://dx.doi.org/10.1016/S0029-7844(02)02076-8

[27] Weber, A.M., Buchsbaum, G.M., Chen, B., et al. (2004) Basic science and translational research in female pelvic floor disorders: proceedings of an NIH-sponsored meeting. Neurourology and Urodynamic, 23, 288-301. 
http://dx.doi.org/10.1002/nau.20048

[28] Gustilo-Ashby, A.M., Lee, U., Vurbic, D., et al. (2010) The impact of cesarean delivery on pelvic floor dysfunction in lysyl oxidase like-1 knockout mice. Female Pelvic Medicine \& Reconstructive Surgery, 16, 21-30.

[29] Wieslander, C.K., Marinis, S.I., Drewes, P.G., Keller, P.W., Acevedo, J.F. and Word, R.A. (2008) Regulation of elastolytic proteases in the mouse vagina during pregnancy, parturition, and puerperium. Biology of Reproduction, 78, 521-528.

http://dx.doi.org/10.1095/biolreprod.107.063024

[30] Budatha, M., Roshanravan, S., Zheng, Q., et al. (2011) Extracellular matrix proteases contribute to progression of pelvic organ prolapse in mice and humans. The Journal of Clinical Investigation, 121, 2048-2059. 